\title{
Quality Assessment of community pharmacy services provided in the United Arab Emirates: patient experience and satisfaction
}

\author{
Farah K. Alhomoud ${ }^{1 *}$, Amr Kunbus ${ }^{2}$, Ahmed Ameer ${ }^{3}$, Faten Alhomoud ${ }^{4}$ \\ ${ }^{1}$ Department of Clinical Pharmacy and Pharmacy Practice, Faculty of Pharmacy, Umm Al-Qura University, Saudi Arabia. ${ }^{2}$ Faculty of Pharmacy, Ajman \\ University of Science and Technology, United Arab Emirates. ${ }^{3}$ Department of Pharmacy, Pharmacology and Postgraduate Medicine, University of \\ Hertfordshire, United Kingdom. ${ }^{4}$ Department of Clinical Pharmacy and Pharmacy Practice, College of Clinical Pharmacy, University of DAMMAM, Saudi \\ Arabia.
}

\section{ARTICLE INFO}

Article history:

Received on: 14/01/2016

Revised on: 24/02/2016

Accepted on: 11/03/2016

Available online: 30/03/2016

Key words:

Patient satisfaction, patient experience, community pharmacy services, United Arab Emirates.

\begin{abstract}
Whilst a limited number of studies have been conducted in nearby Arab countries to evaluate patients' experiences and satisfaction with community pharmacy services provided, in the UAE, this area remains unexamined. Therefore, this study assessed patients' experiences and satisfaction with community pharmacy services in the UAE, which can be used as an indicator to improve services. An online questionnaire was distributed through social media platforms to customers accessing community pharmacies and who are living in the UAE. The study showed that patient satisfaction regarding the two areas, which includes items on communication quality and other aspects of service delivery quality, was more than the average of Likert scale (cut point=3). However, items on satisfaction with consultation quality were a little less than the mentioned cut point. The worst experience, the one with the lowest level of satisfaction, was associated with non-Arabic or non-English speakers and participants over 60 years. The community pharmacy services provided in the UAE are seen to be traditional or product-focused with minimal or negligible pharmaceutical care provided. Therefore, unique structure strategies and health system reforms need to be introduced to follow the trend of extending community pharmacists' roles and to meet the growing needs of patients.
\end{abstract}

\section{INTRODUCTION}

The role of the community pharmacy has been evolving rapidly along with the advancing healthcare system. It has moved from the extemporaneous preparation of medicines and other remedies, and new services have emerged such as providing clinical consultations and working in partnership with other healthcare professionals. Developments in healthcare education, research and technology have played a major role in shaping the current community pharmacy (Bush et al., 2009; McDonald et al., 2010; Scahill et al., 2010; Sadek et al., 2015). These new roles and responsibilities were introduced by the concept of pharmaceutical care, which focuses on patient-centered care and

* Corresponding Author

Farah Kais Alhomoud, Department of Clinical Pharmacy and Pharmacy Practice, Faculty of Pharmacy, Umm Al-Qura University, Saudi Arabia. Email: f_alhomoud83[at]yahoo.com outcomes-oriented practice (Sadek et al., 2015). As a result of this pharmaceutical care concept/strategy, community pharmacists are now integrated into treatment monitoring and providing medication advice, and have a more dominant role in delivering items on the public health agenda, such as smoking cessation and vaccinations (Bryant et al., 2009; Paudyal et al., 2010). Community pharmacists also have an important role in optimizing the use of medications and management of chronic conditions. This will be of an advantage in improving quality of healthcare and help to minimize the overall costs of healthcare in terms of workload and time for other healthcare professionals (Dunlop and Shaw 2002; Smith et al., 2011; Giberson et al., 2015). In addition, these advanced roles and responsibilities have the potential to enhance the professional status of pharmacy practice among other healthcare professionals andincrease the satisfaction of individual pharmacists (Inch et al., 2005; Bryant et al., 2009; Paudyal et al., 2011). 
However, the practice of community pharmacy in the United Arab Emirates (UAE) is still in the process of achieving the concept of pharmaceutical care. The UAE has taken major steps to increase the number of pharmacists and community pharmacies in recent years (Dameh 2009), but the lack of public engagement and possibly awareness is hindering pharmacists from fulfilling their potential in their community practices (WHO 2015). Their practices are still focused on medicine dispensing and other conventional pharmacy services (Haddad et al., 1998; Hasan et al., 2013). However, the quality of the currently provided services in UAE community pharmacies is not known.

Assessment of quality from a patient perspective is a fundamental criterion for evaluating a service and it is a pivotal requirement in quality assessment of healthcare systems in developed countries (Haddad et al., 1998). Patients will view the service provided to them differently to the providers of that service. They can help to indicate areas for improvement within the service and unidentified gaps in practice. Involvement of patients in quality assessment will also provide a measurement or an indication for reaching the objectives of the service (Mehralian et al., 2014). There is evidence to show that satisfied patients are more likely to continue using healthcare services, value and maintain relationships with healthcare providers, participate in their own care, adhere to treatment and have better health outcomes (Aharony and Strasser 1993; Schommer and Kucukarslan 1997). In contrast, neglecting patients' perspectives in the assessment of quality healthcare has been shown to be counterproductive or have undesirable consequences such as patient disconnection, which may lead to non-adherence to medication advice and/or treatment, resulting in treatment failures and waste of health resources (Mehralian et al., 2014).

There are a number of philosophies by which to assess patient experience and satisfaction such as: performance evaluation, disconfirmation of expectation, affect-based assessment and quality-based assessment (Schommer and Kucukarslan 1997). However, performance evaluation is the most often used (Ware et al., 1983). In recent years, patient satisfaction has been assessed in developed countries in relation to advanced pharmaceutical care services or enhanced specific disease management services. However, the findings of these studies are not transferrable/generalizable to the services provided in the UAE community pharmacy since there are many differences between the healthcare systems, patients' characteristics/attributes and nature of the services provided (Hasan et al., 2013).

It is worth mentioning that only a few studies have been conducted in nearby Arab countries to evaluate patients' satisfaction with the provided pharmacy services; these studies took place in Saudi Arabia (Bawazir 2004), Qatar (El Hajj 2011), and Palestine (Khdour and Hallak 2012). In contrast, in the UAE, this area remains unexamined. However, it is important to note that these studies did not use a systematic method to ensure validity and reliability of the quality assessment tool (Hasan et al., 2013), nor did they use an existing validated, reliable and comprehensive assessment tool. However, such a tool is not available in the Arabic language or context of the services provided in the Middle East. Hence, there is a need to develop a quality assessment tool in the Arabic patient's spoken language or adapt an English-dialect questionnaire (Traverso et al., 2007). If the latter alternative is chosen, a rigorous adaptation process must be followed in order to ensure a linguistically and culturally appropriate instrument (Cella et al., 1998; Traverso et al., 2007). This unmet area is exciting since Arabic is the fourth most commonly spoken language in the world and the official language of 19 countries (Ferraro 2002). It is therefore important that adequate tools are presented in Arabic to assess patients' experience and satisfaction with pharmacy services. Thus, this study is aiming to assess patient satisfaction with community pharmacy services delivered in the UAE by developing a valid and reliable quality assessment tool.

\section{MATERIALS AND METHODS}

\section{Quality Assessment Development}

A literature review was undertaken to develop a questionnaire to evaluate patient experience and satisfaction with community pharmacy services. A broad definition of patient satisfaction was used:"a personal evaluation or appraisal of a service or product received" (Donabedian 1988: Aharony and Strasser 1993; Schommer and Kucukarslan 1997; Traverso et al., 2007). This study was based on the performance evaluation philosophy of patient satisfaction assessment. Based on the literature review, a structured questionnaire was devised to assess patient satisfaction with pharmacy services in the UAE. Four community pharmacists were asked to comment on the content and wording of the questions in the tool. This is to ensure that the questionnaire fulfils the requirement of quality assessment domains and is oriented towards the services provided in UAE community pharmacies. The questionnaire was divided into two sections; it included items related to participants' characteristics and items assessing aspects of satisfaction with pharmacy services:

1. Personal data, including age, gender, marital status, education, language, and city of residence.

2. Patient's satisfaction assessment, including items on communication quality, consultation quality and other aspects of service delivery quality. A four-point Likert scale $(1=$ Very poor, 2 = Poor, $3=$ Good, $4=$ Excellent $)$ was used to assess satisfaction with the services.

Closed-ended questions were deliberately chosen for this survey design to ease the capture of responses from participants, and to help to reduce any inadvertent bias in interpreting responses from open-ended questions. The questionnaire then was piloted on four randomly chosen customers in a community pharmacy to assess the feasibility of the questionnaire and to act as a method of face validity. A copy of the tool is available on request.

\section{Questionnaire Translation}

The questionnaire was initially developed in the English language and then translated to Arabic. A three-stage process of 
questionnaire validation was used to ensure reliability of the translation; it involved initial translation by the researcher, group validation and post-validation (Behling and Law 2000). The questionnaire was translated using a parallel blind technique that involved translation of the instrument into the target language (i.e. Arabic) by bilingual speakers $(n=2)$ independently. Both translators are native speakers of Arabic and proficient in English, have health-related postgraduate qualifications, researchinterviewing knowledge and were professionally prepared and acquainted with the objectives of this study. The two drafts were compared and any deviation was addressed. A final validation of the language, content and sensitivity of the questionnaire was assessed by a pharmacy researcher $(n=1)$, pharmacists $(n=4)$ and a group of target language speakers $(n=4)$.

\section{Subjects and Setting}

The study was conducted in the UAE, from March through May 2015. Data were collected using an online questionnaire service. The online questionnaire was distributed through social media platforms including Facebook and Twitter. The inclusion criteria for participants were: all community pharmacy customers (age 15 years old or over), having adequate Arabic or English language proficiency, accessing community pharmacies and living in the UAE. A signed informed consent by parents or legal guardians was needed for participants aged between 15 and 18 years old.

This study was a cross-sectional, survey-based, descriptive/exploratory study. Responses were collected via an online platform (Survey Monkey). An online participant information sheet was provided with the questionnaire to explain the purpose of the study and to obtain consent. Anonymity was assured and no payment or any other equivalent reward was offered to participants.

The sample size was estimated using the Raosoft online calculator (Raosoft) (Raosoft sample size calculator). Total population of the UAE in 2015 was approximately 9.35 million. Therefore, in order to achieve a confidence level of $95 \%$ and a $5 \%$ margin of error, a minimum sample size of 385 was required.

\section{Ethical Approval}

Ethical approval was obtained from Ajman University of Science and Technology Research Ethics Committee.

\section{Data Analysis}

Data were analyzed using SPSS v.22 and Microsoft Excel 2010 software. Descriptive statistics were used to measure the characteristics of the responses. In addition, factor analysis for data reduction was performed to reduce a large number of overlapping variables to a smaller set of factors to evaluate unidimensionality of elements.

The factor analysis was performed using principal component extraction without rotation. Factors with Eigen values greater than one were selected for factor analysis. Items with factor loadings greater than or equal to 0.40 were considered significant.
One sample t-test and one Kruskal-Wallis test for data analysis were used and considered appropriate because the sampling method was simple random sampling, the population was normally distributed when it was tested for normality using Kolmogorov-Smirnov testing, and the sample size was small relative to the population size, which was less than $5 \%$ $(0.0044 \%)$. In regards to the t-test, $\mathrm{p}<0.05$ was set as the level of significance.

\section{RESULTS}

\section{Questionnaire Validity and Reliability}

The questionnaire was developed with reference to the literature relating to community pharmacy services in primary care, and after consultation with researchers and licensed community pharmacists in the UAE. All the views, thoughts and concerns on the proposed study were taken into consideration during the design phase.

This also involved a face and content validity of the quality assessment tool to ensure that the questionnaire is reflective of the community pharmacy practice in the UAE and the services it offers to the public. The validation process was further expanded by piloting the questionnaire with four community pharmacy customers who met the eligibility criteria and who were not aware of this study previously.

According to the results of the factor analysis, the second part of the questionnaire (patients' satisfaction assessment) was divided into three components. These three components comprised communication quality, consultation quality and other aspects of service delivery quality. The result of average variance extraction showed that all components fell into acceptable levels (Table 1)

The reliability of the questionnaire was measured by calculating the Cronbach's alpha value based on questionnaires completed by all the respondents. Based on the literature for confirming reliability, the minimum acceptable value of Cronbach's alpha is 0.5 (Dadfar and Brege 2012). Cronbach's alpha analysis was carried out on the individual sections of the questionnaire along with principal factor analysis.

Therefore, Cronbach's alpha values obtained for questions related to patients' experiences and satisfaction with communication, consultation and other aspects of service delivery quality were equal to $0.97,0.80$ and 0.70 , respectively, which showed a high level of reliability for the three sections. Results of factor analysis and reliability test are shown in Table 1.

The questionnaire was completed by a total of 415 participants of a mean age of 35 years (Range 18 to 68 years old, $\mathrm{SD}=6$ ). Almost an equal number of participants from each gender (Male=221 and Female=194) took part in the questionnaire, the majority are postgraduate or bachelor degree holders $(n=367$; $88.4 \%$ ) and Arabic was reported as the first language by $94 \%$ ( $=$ 391) of the participants. Table 2 presents the key demographic characteristics of the participants. 
Table 1: Results of factor analysis and reliability test.

\begin{tabular}{|c|c|c|c|}
\hline Item & $\begin{array}{l}\text { Factor } \\
\text { Loading }\end{array}$ & $\begin{array}{l}\text { Cronbach's } \\
\text { Alpha }\end{array}$ & AVE* \\
\hline Items on communication quality & & 0.79 & 0.62 \\
\hline Receiving medication with care and full attention & 0.55 & & \\
\hline Observing courtesy and respect from pharmacy staff & 0.43 & & \\
\hline The way the pharmacist answers patients' questions, when asking. & 0.52 & & \\
\hline Allocating time to answer patients' questions & 0.60 & & \\
\hline Allocating time to prepare medication & 0.66 & & \\
\hline Items on consultation quality & & 0.80 & 0.63 \\
\hline Giving explanations on when and how to know if the medication is working (e.g. efficacy) & 0.71 & & \\
\hline Explanation of possible adverse events or side effects (e.g. safety) & 0.60 & & \\
\hline Providing medication counselling and encouraging patients to raise questions regarding medications & 0.68 & & \\
\hline Understanding of what pharmacist say in terms of medication & 0.38 & & \\
\hline Items on other aspect of service delivery quality & & 0.70 & 0.50 \\
\hline Providing printed information or audio-visual patient education materials about drug therapy and/or diseases & 0.56 & & \\
\hline Proper dispensing, labelling and packaging & 0.18 & & \\
\hline Getting help from pharmacy through pharmacy help line service by phone & 0.49 & & \\
\hline $\begin{array}{l}\text { Overall satisfaction regarding community pharmacy services } \\
* \text { Average variance extraction }\end{array}$ & 0.61 & & \\
\hline
\end{tabular}

Table 2: Characteristics of the participants $(\mathrm{N}=415)$.

\begin{tabular}{|c|c|}
\hline Parameter & $\mathrm{N}(\%)$ \\
\hline \multicolumn{2}{|l|}{ Age categories } \\
\hline $15-30$ & $231(55.7 \%)$ \\
\hline $31-45$ & $119(28.7 \%)$ \\
\hline $46-60$ & $60(14.5 \%)$ \\
\hline Above 60 & $5(1.2 \%)$ \\
\hline \multicolumn{2}{|l|}{ Gender } \\
\hline Male & $221(53.3 \%)$ \\
\hline Female & $194(46.7 \%)$ \\
\hline \multicolumn{2}{|l|}{ Educational status } \\
\hline Post graduates or bachelors & $367(88.4 \%)$ \\
\hline Diploma & $35(8.4 \%)$ \\
\hline High school & $13(3.1)$ \\
\hline Illiterate & $0(0 \%)$ \\
\hline \multicolumn{2}{|l|}{ First language } \\
\hline Arabic & $391(94 \%)$ \\
\hline English & $13(3.1 \%)$ \\
\hline Others & $11(3.7 \%)$ \\
\hline
\end{tabular}

\section{Patients' Previous Experiences and Satisfaction with Community Pharmacy Services in the UAE}

Table 3 presents the mean responses to the 13 questions addressing patients' experience and satisfaction with pharmacy services. Table 3 shows that the two components relating to quality of communication and quality of service delivery were more than the cut point (cut point= 3). However, patients' experiences and satisfaction with community pharmacists' quality of communication was less than quality of service delivery. Moreover, community pharmacists' quality of consultation was reported to be slightly below the cut point. The detailed evaluation of the 13-item showed that, among all questions, the good to excellent experience with the highest level of satisfaction related to the 'labelling and packaging services' $(n=3.52)$. The items that related to quality of consultation were associated with the worst experience and lowest level of satisfaction, as shown in Table 3. This can be seen in the 'explanation of all possible adverse events or side effects' $(\mathrm{n}=2.59)$ and 'providing medication counselling and encouraging patients to raise questions regarding medications' $(n=2.84)$. The relationship between categorical variables and patients' experiences and satisfaction was assessed using the KruskalWallis test (Table 4). For the demographic parameters, four variables (age, gender, education status and first language) were evaluated. The findings revealed significantly different levels of experience and satisfaction for different categories of ages. For example, an increase of age correlates to less satisfaction with pharmacy services $(\mathrm{p}=0.001)$. In addition, patients with low educational attainment were unhappy with the communication quality of the service provided $(\mathrm{p}=0.003)$. Additionally, it was found that high patient experience and satisfaction was associated with female patients who are from an Arabic-speaking background and in the middle age ranging from 31 to 45 years old. In contrast, the worst experience and lowest level of satisfaction was associated with non-Arabic or non-English speakers and participants aged over 60 (Table 4). 
Table 3: Descriptive and one sample t test analysis.

\begin{tabular}{|c|c|c|c|}
\hline Item & Mean & $\mathbf{S D}^{\mathbf{a}}$ & $T$-test \\
\hline Items on communication quality & 3.18 & 0.46 & 109.96 \\
\hline Receiving medication with care and full attention & 3.26 & 0.62 & 105.23 \\
\hline Observing courtesy and respect from pharmacy staff & 3.19 & 0.56 & 113.49 \\
\hline The way the pharmacist answers patients' questions, when asking & 3.23 & 0.52 & 124.02 \\
\hline Allocating time to answer patients' questions & 3.08 & 0.64 & 95.33 \\
\hline Allocating time to prepare medication & 3.16 & 0.56 & 111.73 \\
\hline Items on consultation quality & 2.92 & 0.76 & 78.05 \\
\hline Giving explanations on when and how to know if the medication is working (e.g. efficacy) & 2.98 & 0.77 & 76.75 \\
\hline Explanation of all possible adverse events or side effects (e.g. safety) & 2.59 & 0.89 & 58.18 \\
\hline Providing medication counselling and encouraging patients to raise questions regarding medications & 2.84 & 0.81 & 69.80 \\
\hline Understanding of what pharmacist say in terms of medication & 3.27 & 0.61 & 107.47 \\
\hline Items on other aspect of service delivery quality & 3.16 & 0.64 & 103.19 \\
\hline Providing printed information or audio-visual patient education materials about drug therapy and/or diseases & 3.08 & 0.71 & 86.78 \\
\hline Labelling and packaging & 3.52 & 0.50 & 141.47 \\
\hline Getting help from pharmacy through pharmacy help line service by phone & 2.97 & 0.77 & 77.04 \\
\hline Overall satisfaction regarding community pharmacy services & 3.22 & 0.60 & 107.48 \\
\hline \multicolumn{4}{|l|}{${ }^{\mathrm{a}}$ Standard Deviation } \\
\hline${ }^{*}$ Significant at 0.05 & & & \\
\hline
\end{tabular}

Table 4: Participants' characteristics and satisfaction mean rank.

\begin{tabular}{|c|c|c|c|}
\hline \multirow[t]{2}{*}{ Grouping variable } & \multicolumn{3}{|c|}{ Test variables mean rank } \\
\hline & Communication quality & Consultation quality & Other aspect of service delivery quality \\
\hline \multicolumn{4}{|l|}{ Age categories (years) } \\
\hline $15-30$ & 190.07 & 197.58 & 179.01 \\
\hline $31-45$ & 255.05 & 239.87 & 259.25 \\
\hline $46-60$ & 187.47 & 189.28 & 190.90 \\
\hline Above 60 & 149.68 & 157.37 & 117.35 \\
\hline Sig. (2-tailed) & 0.001 & 0.001 & 0.000 \\
\hline \multicolumn{4}{|l|}{ Gender } \\
\hline Male & 192.68 & 194.35 & 192.66 \\
\hline Female & 225.45 & 223.54 & 225.47 \\
\hline Sig. (2-tailed) & 0.09 & 0.28 & 0.27 \\
\hline \multicolumn{4}{|l|}{ Educational status } \\
\hline Post graduates or bachelors & 200.86 & 204.62 & 205.28 \\
\hline Diploma & 240.18 & 227.91 & 230.78 \\
\hline High school & 269.26 & 249.79 & 223.29 \\
\hline Illiterate & $\mathrm{N}$ & $\mathrm{N}$ & $\mathrm{N}$ \\
\hline Sig. (2-tailed) & 0.003 & 0.141 & 0.27 \\
\hline \multicolumn{4}{|l|}{ First language } \\
\hline Arabic & 210.77 & 210.50 & 211.76 \\
\hline English & 146.11 & 179.39 & 149.49 \\
\hline Others & 182.67 & 133.67 & 143.36 \\
\hline $\begin{array}{l}\text { Sig. }(2 \text {-tailed }) \\
* \text { Significant at } 0.05\end{array}$ & 0.05 & 0.09 & 0.07 \\
\hline
\end{tabular}

\section{DISCUSSION}

This study was able to examine the quality of patients' past encounters with community pharmacy services provided in the UAE. This study is believed to be important for the following reasons: firstly, a newly validated and reliable tool was developed and used for the first time in the Middle East to assess patients' previous experiences and satisfaction with community pharmacy services in the Arabic context. The developed tool can be used in different Arab countries, as studies have shown that the practice of pharmacy is similar in Arab countries (Al-Wazaify and AlbsoulYounes 2005; Kheir et al., 2009; Sweileh et al., 2009; Awad and Abahussain 2010). Therefore, the findings of this study can be used to improve service provision in many Arabic countries, which could eventually result in meeting patients' needs and therefore optimal outcomes. Secondly, considering patients' previous experiences and satisfaction is very important to improve the quality of health services, health promotion and reduction of health costs by determining patients' needs, priorities and concerns. In contrast, excluding patients from the assessment of quality healthcare may result in them being unwilling to access the healthcare system or follow the medical advice they are given, due to being not involved or dissatisfied with the healthcare services, which may lead to suboptimal outcomes or treatment failures. This will also have an effect on patients' adherence, concordance and compliance.

Using the tool developed in this study, it was possible to evaluate the quality of communication, consultation and the overall quality of pharmaceutical service delivery in a UAE community pharmacy. The study found that patients reported worst experience and lowest level of satisfaction in relation to the quality of consultation provided by community pharmacists (2.92 \pm 0.76 ). This is consistent with the findings of similar studies, both conducted in Iran (Dadfar and Brege 2012; Mehralian et al., 2014), in which the lowest level of satisfaction was related to the consultation services. In contrast, the greatest experience and the 
highest level of satisfaction related to quality of communication by community pharmacists $(3.18 \pm 0.46)$. This is also in agreement with past studies, one carried out in the USA in 2002 (Larson et al., 2002) and the other in Iran in 2014 (Mehralian et al., 2014).

\section{Quality of Communication}

In addition, the quality of communication was assessed. It was found that the greatest experience and highest level of satisfaction was reported by patients when 'receiving medication with care and full attention' $(n=3.26)$ and 'the way the pharmacist answers patients' questions, when asking $(n=3.23)$, whilst 'allocating time to answer patients' questions' and 'allocating time to prepare medications' were associated with the lowest level of satisfaction by participants. However, in a study of 1,144 consumers of 55 community pharmacies in Saudi Arabia, it was found that $44.8 \%$ of participants felt that pharmacists in Saudi Arabia had given them enough time to discuss their issues completely and had listened to their health-related issues (Bawazir 2004). Furthermore, in another cross-sectional descriptive study, conducted in Iran by Mehralian et al., (Mehralian et al., 2014) amongst 800 patients of 200 community pharmacies, it was reported that the highest satisfaction level was mainly related to two domains, 'observe courtesy and respect' and 'reliability of pharmacists' comments', whereas satisfaction with 'allocation of sufficient time to respond to clients' questions' was reported as high or very highly satisfactory by $37.4 \%$ of the participants.

\section{Quality of Consultations}

As mentioned above, the study also examined the quality of consultations provided in UAE community pharmacies. It was found that the worst patients' experience and the lowest level of satisfaction is associated with 'explanation of possible adverse events or side effects' $(n=2.59)$ and 'providing medication counselling and encouraging patients to raise questions regarding medications' $(n=2.84)$. These findings are consistent with a study conducted in the UAE by Hassan et al., in 2013, who reported that participants gave low scores to the particular counselling they received about their medication and how it works (41\%), side effects (16\%), and the interest shown by community pharmacists in helping them make best use of their medication (30\%) (Hasan et al., 2013). In this study, some participants had undergone an unpleasant experience and were disappointed with the consultation services provided by community pharmacists in the UAE. This could be due to the fact that specific counselling is not a routine occurrence/procedure in community pharmacies in the UAE (Hasan et al., 2013). Therefore, it is recommended to conduct larger-scale quantitative studies to examine the extent and nature of counselling in the UAE community pharmacy. This will aid the development of an intervention to improve the quality of community pharmacies in the UAE and also improve patient safety, medication adherence, concordance and compliance with prescriptions.

\section{Future Recommendations}

The findings of this study provide a number of service improvement opportunities. As was illustrated, there is a need to improve the education and training of pharmacists in the UAE. Pharmacists need to be equipped with vital communication and consultation skills to be able to provide a safe clinical service to a multi-cultured and diverse population. This education and training should also extend to practicing pharmacists and other pharmacy staff in the form of regular continuous professional development (CPD). This is also to take into account that the UAE has a large number of expatriate pharmacists who have various levels of background, experience and training (Hasan et al., 2013). This quality analysis of the community pharmacy provision in the UAE also found there is a need to improve and/or develop the following areas: need for better pharmacy operating procedures to maximize the time spent in patient consultation and minimize time in preparation/assembly of prescription items, and engagement of pharmacists in more clinical roles such as medicine use reviews (MURs), health assessment, monitoring, and screening of chronic diseases, repeat dispensing, and public health promotion and awareness (e.g. smoking cessation).

\section{Study Strengths}

The findings of this study could be used for service development/improvement by pharmacy commissioners, pharmacy managers, educators and individual pharmacists. As mentioned previously, this is one of the first studies in the UAE to evaluate the quality of the community pharmacy service from a patient's perspective. A thorough method was used to develop, validate, implement and analyze a short questionnaire to capture UAE community pharmacy users' opinions on the quality of the service they have received. This questionnaire has utilized the increasingly popular method of communicating in the Middle East, which is online social media platforms.

\section{Study Limitations}

The interpretation of the findings must also be considered along with the study limitations. This study used a direct selfcompletion method (e.g. online questionnaire) due to its simplicity and feasibility for most settings. However, it could be subjected to memory bias or misunderstanding of the questions, which may lead to data inaccuracies. Additionally, the study may not have captured the ageing population of the UAE, who are among the most frequent users of community pharmacies. Conducting faceto-face interviews or handing out paper questionnaires at the community pharmacies could have avoided this limitation. Moreover, the study could not verify if the responses were directly relating to a patient's experience in a community pharmacy or if there was an overlap with a hospital pharmacy service provided to the patient, since this a retrospective analysis of previous encounters. Again, this could have been reduced by conducting direct data collection from patients in different community pharmacies. 


\section{CONCLUSION}

The community pharmacy services provided in the UAE are seen to be traditional or product-focused with minimal or negligible pharmaceutical care provided. Therefore, unique structure strategies and health system reforms need to be introduced to the community pharmacy profession to follow the trend of extending community pharmacists' roles and to meet the growing needs of patients, which can improve the quality care and optimize the use of medication. Moreover, in order to explore services available for patients in the UAE and provide more evidence-based interventions, further studies are required to examine the extended roles of community pharmacists.

\section{ACKNOWLEDGEMENTS}

We thank Ms. Sukaina A. Damji for her assistance in proofreading and editing the article, which helped the readability of our work.

\section{REFERENCES}

Aharony L, Strasser S. Patient satisfaction: what we know about and what we still need to explore. Med Care Rev, 1993; 50: 49-79.

Awad A and Abahussain E. Health promotion and education activities in community pharmacists in Kuwait. Pharm World Sci, 2010; 32: $146-153$.

Al-Wazaify M and Albsoul-Younes A. Pharmacy in Jordan. Am J Health Syst Pharm, 2005; 62: 2548-2551.

Bawazir SA. Consumer attitudes towards community pharmacy services in Saudi Arabia. Int J Pharm Pract, 2004; 12: 83-9.

Behling O, Law K. 2000. Translating questionnaires and other research instruments: problems and solutions. London: Sage Publication.

Bryant LJ, Coster G, Gamble GD, et al. General practitioners' and pharmacists' perceptions of the role of community pharmacists in delivering clinical services. Res Social Admin Pharm, 2009; 5: 347-362.

Bush J, Langley CA, Wilson. The corporization of community pharmacy: implications for service provision, the public health function, and pharmacy's claims to professional status in the United Kingdom. Res Social Admin Pharm, 2009; 5: 305-318

Cella D, Hernandez L, Bonomi A, et al. Spanish language translation and initial validation of the functional assessment of cancer therapy quality of life instrument. Med Care, 1998 36: 1407-18.

Dadfar $\mathrm{H}$ and Brege $\mathrm{S}$. Differentiation by improving quality of services at the last touch point: the case of Tehran pharmacies. Int J Qual Serv Sci, 2012; 4: 345-63.

Dameh M. Pharmacy in the United Arab Emirates. Southern Med Review,2009; 1: 15-18.

Donabedian A. The quality of care: how can it be assessed? J Am Med Assoc, 1988; 260: 1743-1748.

Dunlop JA and Shaw JP. Community pharmacists' perspectives on pharmaceutical care implementation in New Zealand. Pharm World Sci, 2002;24: 224-230.

El Hajj M, Salem S, Mansoor H. Public's attitudes towards community pharmacy in Qatar: a pilot study. Pat Pref Adher, 2011; 5: 405-422.

Ferraro FR. 2002. Minority and Cross-cultural Aspects of Neuropsychological Assessment: Studies on Neuropsychology, Neurology and Cognition. Lisse, NL: Swets and Zeitlinger.

Giberson S, Yoder S, Lee MP. (2011) Improving patients and health system outcomes through advanced pharmacy practice. A report to the U.S. Surgeon General. USA: Office of the Chief Pharmacist. U.S. Public Health Service [ONLINE] Available at: http://www.accp.com/docs/positions/misc/Improving_Patient_and_Health _System_Outcomes.pdf. [Accessed 29 September 2015].
Haddad S, Fournier P, Potvin L. Measuring lay people's perceptions of the quality of primary health care services in developing countries. Validation of a 20-item scale. Int J Qual Health Care, 1998; 10: 93-104.

Hasan S, Sulieman H, Stewart K, et al. Assessing patient satisfaction with community pharmacy in the UAE using a newly validated tool. Res Social Adm Pharm, 2013; 9: 841-850.

Inch JM, Bond CM, Lee AJ. Scottish community pharmacists' current involvement in and attitudes towards 'extended service' provision: a national survey. Int J Pharm Practice, 2005; 13: 289-301.

Khdour MR and Hallak HO. Societal perspectives on community pharmacy services in West Bank - Palestine. Pharm Pract, 2012 10: 17-24

Kheir N, Zaidan M, Younes H, et al (2009) Pharmacy education and practice in 13 middle eastern countries. Am J Pharm Educ 72: 133.

Larson LN, Rovers JP, MacKeigan LD. Patient satisfaction with pharmaceutical care: update of a validated instrument. J Am Pharm Assoc Wash, 2002; 42: 44-50.

McDonald R, Cheraghi-Sohi S, Sanders C, et al. Professional status in a changing world: the case of medicine use reviews in English community pharmacy. Social Sci Med, 2010; 71: 451- 458.

Mehralian G, Rangchian M, Rasekh HR. Client priorities and satisfaction with community pharmacies: the situation in Tehran. Int J Clin Social Sci Med Pharm, 2014; 36: 707-715.

Paudyal V, Hansford D, Scott Cunningham IT, et al. Crosssectional survey of community pharmacists' views of the electronic MAS in Scotland. Int J Pharm Practice, 2010; 18: 194-201.

Paudyal V, Hansford D, Cunningham S, et al. Pharmacy assisted patient self-care of minor ailments: a chronological review of UK health policy documents and key events 1997-2010. Health policy, 2011; 101: 253-259.

Raosoft sample size calculator. Raosoft [ONLINE] Avialable at:http://www.raosoft.com/samplesize.html. [Accessed 20 September 2015].

Sadek MM, Elnour AA, Al Kalbani NM, et al. Community pharmacy and the extended community pharmacist practice roles: The UAE experiences. Saudi Pharm J. 2015. http://dx.doi.org/10.1016/ j.jsps.2015.03.023.

Scahill S, Harrison J, Sheridan J. Pharmacy under the spotlight: New Zealand pharmacists' perceptions of current and future roles and need for accreditation. Int J Pharm Practice, 2010; 18: 59-62.

Schommer JC, Kucukarslan SN. Measuring patient satisfaction with pharmaceutical care services. Am J Health Syst Pharm, 1997; 54 2721-2732.

Smith M, Giuliano MR, Starkowski MP. In connecticut: improving patient medication management in primary care. Health Affairs, 2011; 30: 646-654

Sweileh W, Al Jabi S, Sawalha A. Pharmacy education and practice in West Bank, Palestine. Am J Pharm Educ, 2009; 73: 38.

Traverso M, Salamano M, Botta C et al. Questionnaire to assess patient satisfaction with pharmaceutical care in Spanish language. Int J Qual Health Care, 2007; 19: 217-224.

Ware J, Snyder MK, Wright WR, Davies AR. Defining and measuring patient satisfaction with medical care. Eval Prog Plan, 1983, 6 247-263.

World Health Organization (WHO). Management quality assurance Care [ONLINE]. Available at: http://www.who.int/management/ quality/assurance/QualityCare_B.Def.pdf. [Accessed 26 September 2015].

How to cite this article:

Alhomoud FK, Kunbus A, Ameer A, Alhomoud F. Quality Assessment of community pharmacy services provided in the United Arab Emirates: patient experience and satisfaction. J App Pharm Sci, 2016; 6 (03): 017-023. 